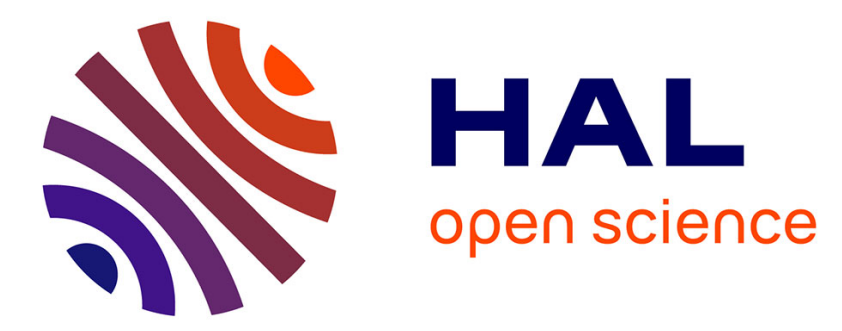

\title{
Le passé et le présent des politiques d'urbanisation et de logement en Turquie
}

\author{
Gülçin Erdi
}

\section{To cite this version:}

Gülçin Erdi. Le passé et le présent des politiques d'urbanisation et de logement en Turquie. Naqd, Revue d'études et de critique sociale, 2020, Les politiques de logement social au Maghreb/Machrek et dans le Sud Global, 38-39 (1-2), pp.51-64. 10.3917/naqd.038.0051 . halshs-03479632

\section{HAL Id: halshs-03479632 \\ https://shs.hal.science/halshs-03479632}

Submitted on 14 Dec 2021

HAL is a multi-disciplinary open access archive for the deposit and dissemination of scientific research documents, whether they are published or not. The documents may come from teaching and research institutions in France or abroad, or from public or private research centers.
L'archive ouverte pluridisciplinaire HAL, est destinée au dépôt et à la diffusion de documents scientifiques de niveau recherche, publiés ou non, émanant des établissements d'enseignement et de recherche français ou étrangers, des laboratoires publics ou privés. 


\title{
Le passé et le présent des politiques d'urbanisation et de logement en Turquie
}

\author{
Gülçin Erdi*
}

L'urbanisation en Turquie peut s'expliquer à travers trois phases historiques : la période avant 1980, la période entre 1980 et 2001 et la période après 2001. La première phase correspond au début de l'urbanisation dès les années 1930, et est clairement liée à l'intensification de l'exode rural (Öncü 1988). Elle est marquée par l'absence de politiques publiques en matière de logement. De fait, ce n'est que très tardivement, dans le courant des années 1980, que de telles politiques ont vu le jour, aux fins de la construction de logements collectifs avec la création de l'Administration des logements collectifs (TOKI en turc). Cependant, on ne peut pas parler en Turquie de l'existence de logements sociaux de type HLM, comme en France. Il existe des programmes d'accession à propriété pour des revenus modestes, mais pas de location à des prix indexés au revenu des individus.

L'absence de ces politiques a été à l'origine de très grandes difficultés d'installation en ville pour les populations rurales, arrivées en masse dès les années 1950, qui ont dû mobiliser toutes leurs compétences et leurs réseaux pour faire face à leur besoin de logement. Karpat (1976) souligne que la population urbaine des villes de Turquie de 10000 habitants ou plus a augmenté de $409 \%$ de 1927 à 1965. Par exemple, à Ankara, la population a été multipliée par 70 entre 1927 et 2015, passant de 74553 en 1927 à 5,27 millions en 2015 (TUIK, 2015). De ce fait, une des solutions que ces migrants mirent en œuvre fut le recours à des constructions informelles, appelées gecekond $u^{1}$ en Turquie, le terme rendant compte de la rapidité et de la clandestinité de l'opération. Très vite, au cours des années 1950, les gecekondus constitueront des éléments essentiels du processus d'urbanisation.

A partir des années 1980, les gecekondus deviennent un élément structurel de l'urbanisation et on entre dans une phase d'acceptation et de légalisation de ces habitations par les décideurs publics. Ce tournant est en large partie dû à la croissance exponentielle de leur part dans le parc de logements urbains et à celle de leurs populations au sein des villes, faisant en sorte qu'elles constituent désormais un enjeu politique et électoral majeur pour les gouvernements successifs. D'après Keleş (1992), dans les années 90, 72\% de la population urbaine d'Ankara vivaient dans des gecekondus (p.215).

Au cours des années 1980 et 1990, les gecekondus deviennent une ressource immobilière pour leurs propriétaires. Nombre d'entre eux décident de léguer leur gecekondu et son terrain à des promoteurs immobiliers en échange de 2 ou 3 appartements dans l'immeuble qui sera construit sur son emplacement. Ceci permet aux habitants d'augmenter leur niveau de vie en accédant à des logements régularisés et favorise une transformation progressive de ces quartiers. Avec l'introduction de règles économiques néolibérales dans la deuxième moitié des

\footnotetext{
* Chercheuse au CNRS / UMR CITERES (Univ. de Tours)

${ }^{1}$ Le terme est constitué de deux mots qui signifient, l'un, la « nuit » et l'autre, « posé ». Il désigne donc un habitat « posé en une nuit ».
} 
années 1980 sous le pouvoir du Parti de la mère-patrie (ANAP, droite libérale), le gouvernement turc de Turgut Özal s'engage dans une véritable thérapie de choc, comme ses homologues l'ont fait ailleurs en Europe, accompagnée d'une stratégie de croissance axée sur les exportations (Bekmen 2014: 54). Entre 1980 et 2001, le développement économique domine la politique publique de la Turquie et conduit à l'émergence de grands projets de développement tels que le projet de l'Anatolie du Sud-Est $(\mathrm{GAP})^{2}$. Cette époque a également été associée au développement de coopératives de construction et à l'introduction de très gros entrepreneurs de la construction sur le marché du logement. Au cours de cette période, de grands consortiums immobiliers ont commencé à influencer le marché du logement, et ils ont identifié les zones de gecekondus des centres-villes comme des sites potentiellement rentables.

Avant l'arrivée au pouvoir du Parti de la Justice et du Développement (AKP), les gouvernements précédents n'avaient pas tenté de transformer les zones de gecekondus d'une manière massive, craignant une réaction des électeurs et une défaite électorale subséquente. Cette position a évolué à la suite de deux événements majeurs survenus en Turquie, le premier étant le grand tremblement de terre de 1999 dans la mer de Marmara près d'Istanbul, qui a causé la mort de 16000 personnes et la destruction de 20000 bâtiments. Le second est la crise financière de 2001 qui a provoqué une rupture politique (les partis politiques habituels n'ont pas eu d'élus au Parlement, par exemple) et économique considérable, débouchant sur la victoire électorale de l'AKP.

Dans mon article, je propose notamment de focaliser sur l'urbanisation au prisme du besoin de logement et de la posture des décideurs publiques sous le pouvoir de l'AKP dans les années 2000. Des pistes de réflexions sur l'absence des politiques publiques de logement et la résilience des migrants ruraux dans leur vie urbaine vis-à-vis de leur vulnérabilité résidentielle seront proposés. Pour ce faire, je propose d'étudier le cas de deux grandes métropoles de la Turquie, Istanbul et Ankara pour montrer à la fois les éléments similaires de leur urbanisation et leurs différences liées à leurs positionnement sociaux, économiques et politiques respectifs dans l'histoire de l'urbanisation du pays.

\section{Les années 2000 : un tournant dans l'urbanisation des métropoles en Turquie}

Depuis le début des années 2000, un certain nombre de contestations internationales, telles que des mouvements altermondialistes, ont vu le jour en réaction à la restructuration néolibérale et à l'érosion progressive des droits des citoyens. Ces mouvements qui contestent les impacts de la mondialisation néolibérale ont progressivement commencé à se concentrer sur les villes et à scruter la gouvernance urbaine actuelle, la précarisation croissante et la ségrégation socio-spatiale au sein des villes. Lors du Forum social européen qui s'est tenu à Istanbul en 2010, un grand nombre de panels sur le thème du droit à la ville ont réuni des militants et des habitants de différents quartiers détruits ou menacés de destruction en raison des vastes programmes de transformation urbaine, pour discuter de la possibilité de créer un mouvement urbain commun. Par ailleurs, deux manifestations ont été organisées avec des participants internationaux dans les quartiers Tozkoparan et Sarıer à Istanbul, afin de protester

\footnotetext{
${ }^{2}$ Ce programme de développement régional, lancé dans les années 1980 au sud-est de la Turquie, était composé de plusieurs projets dans le domaine de l'agriculture et de l'énergie incluant la construction de 11 barrages hydroélectriques sur le Tigre et l'Euphrate, des canaux d'irrigation et des systèmes d'arrosage dans toute la région. L'objectif était à la fois d'accélérer le développement de la région la moins développée de la Turquie, peuplée à forte majorité par des Kurdes, et de de barrer la route aux séparatistes kurdes du PKK.
} 
contre des projets menaçant les habitants d'expulsion et de réinstallation dans des conditions dégradées.

L'ultime rejet de ces projets a été la mobilisation nationale en 2013 contre la démolition d'un grand parc urbain (le Parc Gezi), le seul dans le quartier Taksim-Beyoğlu, que le gouvernement voulait remplacer par un centre commercial et une résidence sous forme de la réplique d'une ancienne caserne militaire ottoman (Erdi 2019).

Ces mobilisations que l'on a observées tout au long des années 2000 sont révélatrices des évolutions et transformations des politiques d'urbanisation en Turquie, qui vont de pair avec l'évolution du système politique dans sa globalité.

Élu après la crise économique de 2001, le gouvernement de l'AKP a introduit de nouvelles mesures néolibérales afin de surmonter les problèmes économiques et financiers auxquels le pays était confronté et de relancer la croissance économique. La stratégie économique néolibérale de l'AKP présentait deux caractéristiques principales. La première était le soutien aux groupes capitalistes islamiques proches du parti afin de consolider le bloc du pouvoir assurant une unité et un lien entre la classe politique et les groupes capitalistes (Erdi 2016). Le second était la promotion de projets d'urbanisme et de développement en boostant le secteur de la construction, déjà dynamisé dans les années 1990 via l'émergence des consortiums immobiliers et la vente d'un certain nombre de bâtiments publics et de terrains urbains. Le gouvernement a désigné le secteur comme une solution pour relancer l'économie du pays et stimuler la croissance, ainsi que pour assurer la montée en puissance d'une nouvelle classe capitaliste proche de son idéologie conservatrice et de ses objectifs politiques (Gürek 2008).

Sous le règne de l'AKP, les villes de Turquie sont donc devenues centrales pour la reproduction continue du néolibéralisme lui-même, constituant des cibles géographiques et des laboratoires de plus en plus importants pour une variété d'expériences de politique néolibérale visant à accroître la valeur foncière du sol (Erdi-Lelandais 2016). Celles-ci incluent la création de centres financiers, la construction de centres commerciaux et d'attractions touristiques, et la construction de gated communities de luxe. Certains projets récents, comme la construction d'un nouveau palais présidentiel à Ankara, le troisième aéroport ou Kanal Istanbul ${ }^{3}$, ainsi que des vastes projets de transformation urbaine dans des quartiers informels ou insalubres comme la Vallée de Dikmen à Ankara ou Sulukule à Istanbul reflètent certaines caractéristiques communes de ce régime urbain néolibéral qui pourraient se résumer comme suit :

(i) L'État apparaît comme la principale force d'impulsion et le promoteur majeur via la mobilisation des compétences politico-économiques de diverses institutions publiques et la mise en place d'un nouveau cadre juridique sur la propriété et l'urbanisation ;

(ii) La transformation urbaine et les projets de développement (PD) à grande échelle sont les principaux outils de restructuration des villes ;

(iii) La réalisation ultérieure de la rente foncière incorporée dans l'environnement nouvellement construit de la ville est l'un des principaux objectifs ;

(iv) La transformation urbaine et les PD sont considérés comme des catalyseurs de la croissance économique et de la création d'une nouvelle classe capitaliste conservatrice proche du gouvernement ;

\footnotetext{
${ }^{3}$ Ce projet consiste en la construction d'un canal navigable, situé sur la rive européenne et destiné à désengorger le Bosphore. Le projet, lancé en 2011, consisterait à relier la mer Noire et la mer de Marmara. Longueur du canal : $47 \mathrm{~km}$, largeur : 100 à $150 \mathrm{~m}$; profondeur : $25 \mathrm{~m}$.
} 
(v) Le processus de prise de décision et de réalisation des projets urbains est fermé à la discussion démocratique délibérative incluant les habitants ; il est autoritaire, sécuritaire et imposé du haut vers le bas.

Ces caractéristiques sont facilement démontrables dans plusieurs actes du gouvernement. En mettant en œuvre un certain nombre de réformes juridiques et institutionnelles, l'AKP a décidé de restructurer la gouvernance des marchés immobiliers turcs et l'urbanisme sous le contrôle de l'État, avec des conséquences importantes pour la géographie socio-économique des villes et l'environnement rural. La première étape de cette restructuration a été le renforcement des compétences de l'Administration des Logements Collectifs (TOKI) en 2003 par la loi ${ }^{\circ}$ 4966, permettant le transfert de tous les terrains du Domaine public à son usage avec l'autorisation directe du Premier ministre afin d'y construire des logements. Entre 2004 et 2008, plusieurs lois ont été adoptées par le Parlement afin de définir les orientations de la politique foncière et du logement. La loi $\mathrm{n}^{\circ} 5162^{4}$, votée en mai 2004, a donné à TOKI le pouvoir de procéder aux expropriations dans les zones de rénovation urbaine, d'établir des partenariats avec des entreprises privées et des trusts financiers et de développer des projets de transformation dans les zones de gecekondus. Une autre loi importante (loi ${ }^{\circ}{ }^{\circ} 5366,5 / 2005$ ) accorde aux communes et au TOKI la possibilité de réaliser des projets de rénovation urbaine non seulement dans des zones d'habitat informel, mais aussi dans des quartiers historiques jugés délabrés dans l'objectif de leur revalorisation et réutilisation. En 2007, par la loi n ${ }^{\circ} 5609$, le TOKI est devenue la seule autorité dans la détermination des zones de construction et la vente des terrains publics.

Enfin, en 2012, avec la « loi sur la transformation des zones à risques de catastrophe » (loi $\mathrm{n}^{\circ}$ 6306), le gouvernement a eu les mains libres pour entreprendre des projets de renouvellement à travers le pays sous la gestion du TOKI, en utilisant l'argument du « risque ». À cet égard, «les propriétaires des logements situés dans des zones déclarées à risque sont obligés de les vendre à la municipalité ou de les démolir à leurs propres frais. En fonction de la solidité financière du propriétaire, ce dernier peut s'insérer dans un projet de même taille ou plus petit, plus proche ou plus éloigné de l'emplacement d'origine. De plus, les droits des locataires sont totalement ignorés dans le processus » (Çavuşoğlu et Strutz 2014, p.147).

Comme le montre l'échantillon de lois que nous venons d'évoquer, le rôle du TOKI est désormais indéniable dans les politiques de logement, étant donné qu'il centralise toutes les décisions en matière de construction de logement. Auparavant institution publique à but non lucratif pour le logement collectif, le TOKI a aujourd'hui l'autorisation d'entreprendre des projets de logement «à but lucratif» sur des terrains publics, soit par l'intermédiaire de ses filiales, soit par le biais de partenariats public-privé, le tout pour lever des fonds pour la construction présumée de logements sociaux. Cependant, en 2012, dans le nombre total de projets relevant de la gestion du TOKI, les projets de logements à prix modéré ne représentaient que $6,51 \%$ contre $75,25 \%$ de logements à but lucratif (Erdi-Lelandais 2014). Le TOKI est donc devenu un instrument qui génère du capital grâce à la fois au développement de projets dans des zones à profit foncier élevé ou en devenir, par le biais des projets de construction mis en place avec des entreprises de construction sous-traitantes comme Kuzu, Biat, Aksa, Ağaoğlu, Taşyap1, Albayrak, İhlas et Çalık (Gürek 2008; Çavuşoğlu et Strutz 2014), toutes proches du

\footnotetext{
${ }^{4}$ Les lois citées ne constituent pas une liste exhaustive. La législation sur l'urbanisation constitue un vaste chantier en Turquie et nous ne citons que quelques exemples significatifs en la matière.
} 
gouvernement et de la famille du président de la République, Recep Tayyip Erdoğan. Ces changements ont assuré la néo-libéralisation à la fois du foncier et du régime du logement en Turquie. Dans tous les projets confiés à ces entreprises, l'État s'engage également à partager les risques et à indemniser les entreprises en cas de soucis. Par exemple, pour la construction du troisième aéroport d'Istanbul, l'État a fourni une garantie assurant la rentabilité de l'investissement et de la gestion future au consortium ayant remporté l'appel d'offres de sa construction et de sa gestion ${ }^{5}$. L'Etat prend en outre en charge, au travers du Trésor public, les intérêts des emprunts levés sur le marché international des capitaux par ce consortium ${ }^{6}$.

Dans ce régime néolibéral, la transformation urbaine est considérée comme un outil majeur pour maximiser la production de la rente foncière, grâce à la marchandisation de superficies accrues que favorise la reconfiguration de l'espace de la ville en fonction de sa valeur d'échange et non d'usage. Par conséquent, le premier objectif est de transformer les gecekondus situés à proximité du centre-ville, parce qu'ils ont des valeurs immobilières élevées. L'objectif de l'urbanisme est désormais de créer des villes dont les pôles sont réservés aux affaires, au tourisme et aux loisirs (centres commerciaux, résidences, parcs d'attractions, musées, etc.), où un type d'habitat et de mode de vie s'impose sans prendre en compte les caractéristiques culturelles, régionales et environnementales, ni les modes de vie de différentes populations (Dinçer 2011). Les quartiers populaires habités par les plus pauvres de la ville, dont la valeur foncière possède le plus grand potentiel d'augmentation, ont été remodelés par des opérations conçues dans le cadre de partenariats locaux entre les municipalités et le secteur privé ; les logements ainsi produits ont été attribués principalement à de nouveaux habitants d'Istanbul : des personnes à haut capital culturel et économique ( locaux ou étrangers), des cadres travaillant dans des secteurs très demandés à l'ère postindustrielle (par exemple, la finance, le design et l'informatique), ainsi que des professionnels dans les domaines institutionnalisés des arts et de la culture (Adanali et.al 2009).

Ce modèle d'urbanisme, étudié maintes fois par de nombreux universitaires (Smith 1979, 1996 ; Zukin 1987 ; Florida 2002,2017 ; Lees et.al 2010), replace la ville dans un nouveau rôle. Il n'est plus question de la concevoir comme un espace créateur de liens et offrant l'opportunité de brassages sociaux, mais plutôt comme un espace susceptible d'avoir une utilité financière pour l'accumulation du capital. De ce fait, les projets urbains, y compris ceux qui concernent le logement, sont souvent conçus de manière totalement extérieure à une quelconque politique publique de logement à destination des populations affectées par ces projets, notamment celles des gecekondus pour lesquelles la transformation ou la rénovation de leur quartier implique à chaque fois la démolition de leur logement et leur déplacement plus ou moins forcé dans des quartiers périphériques qui ne relèvent pas de leur préférence individuelle.

\footnotetext{
${ }^{5}$ L'Etat garantit au consortium un volume minimal de passagers chaque année, lesquelles assurent à ce dernier une partie de ses recettes. Si ce chiffre n'est pas atteint, l'Etat verse $20 €$ par passager « manquant» (différence entre le nombre garanti et le nombre réel de passagers). Ce type d'accord n'est pas propre à ce projet d'aéroport, mais caractérise pratiquement tous les projets de développement (ponts, autoroutes, etc.).

${ }^{6}$ Ces informations sont tirées du rapport préparé par la Défense des Forêts du Nord d'Istanbul, une plate-forme civile créée pour s'opposer à la construction du troisième aéroport. Rapport (en turc) disponible sur: http://www.kuzeyormanlari.org/2014/03/21/kuzey-ormanlari-savunmasi-3-havalimani-raporu-nereden-baksankatliam-yagma-saibe-guncellendi-3-2-2014/
} 
Je me propose donc maintenant de discuter de la place du logement dans l'urbanisation actuelle en Turquie et de montrer ce que l'absence des véritables politiques de logement génère comme vulnérabilité résidentielle pour des habitants des grandes métropoles du pays.

\section{Politiques de logements, urbanisation et vulnérabilités résidentielles dans la Turquie d'aujourd'hui}

Comme nous l'avons mentionné dans la première partie de cet article, l'accès au logement, en Turquie, a longtemps relevé de la seule initiative individuelle. Quand, du fait de l'urbanisation rapide, le problème a revêtu une ampleur considérable et est devenu de plus en plus aigu, les pouvoirs publics se sont avérés incapables de produire des solutions efficaces. De fait, ce n'est que très tardivement, dans le courant des années 1980, que des politiques publiques ont vu le jour via la création du TOKI aux fins de la construction de logements collectifs. Ces politiques visaient à fournir des logements à des classes sociales modestes par un dispositif d'accession à la propriété par le biais de coopératives de construction ou de crédits à taux faible, ce qu'a rendu possible la transformation, en 1988, d'une banque publique, fondée en 1926 par Atatürk, en Emlak Bankast (Banque de l'Immobilier). Les limites de ces politiques de logement étaient leur focalisation sur la propriété privée, ce qui leur faisait négliger le besoin locatif. Cette posture idéologique explique l'absence de volonté d'investir dans la construction de logements sociaux pour les classes pauvres, incapables qu'elles étaient d'entrer dans des programmes d'accession à la propriété. Cela constitue l'une des raisons majeures de la multiplication des gecekondus dans les grandes métropoles.

En d'autres termes, des migrants ruraux qui sont venus dans les métropoles soit pour des raisons économiques soit pour des raisons politiques, notamment dans le cas des Kurdes en raison du conflit au sud-est du pays, n'avaient d'autres choix que de subir les aléas liés à leur installation dans la ville sans être en mesure $d$ ' « inventer » une quelconque solution ou de concevoir des adaptations à leur situation. Cependant, en partant de la théorie de Genard (2014) sur la vulnérabilité, on peut suggérer qu'ils sont «potentiellement fragiles, susceptibles de verser dans l'hétéronomie [...], mais toujours aussi susceptibles de se reprendre, de se ressaisir, [car] dispos[a]nt toujours des capacités minimales sur lesquelles s'appuyer pour retrouver davantage d'autonomie » (p.42-44). Les analyses que nous avons effectuées dans deux quartiers de gecekondus, l'un situé à Istanbul et l'autre à Ankara, confirment l'hypothèse que les nouveaux arrivants parviennent assez rapidement à construire un logement, à s'y établir de manière durable, faisant ainsi la preuve de leur capacité à s'adapter à l'environnement changeant qu'est la grande ville et aux obstacles qu'elle dresse devant eux (Brodiez-Dolino, 2014). L'absence de politiques du logement a fait également émerger des réseaux informels assurant, au bout du compte, la distribution des terrains, dans l'objectif de construire des gecekondus. Tantôt (et le plus souvent), il s'agissait de groupes mafieux qui parcellisaient des terrains publics et parfois c'étaient des groupes politiques de gauche qui distribuaient des terrains avec un projet révolutionnaire, celui de constituer des zones autonomes et autogérées, qui seraient acquises de ce fait à la cause socialiste. C'est ainsi qu'ont été créés le quartier du $1^{\text {er }}$ Mai à Istanbul et le quartier de la Vallée de Dikmen à Ankara.

Selon le récit de leurs habitants, les premières installations y ont été orchestrées par des groupes immobiliers mafieux qui vendaient des parcelles de terrains publics aux migrants ruraux afin qu'ils puissent construire leur gecekondu (Aslan, 2004). Le contrôle de la distribution de ces terrains est progressivement passé aux mains de groupes se réclamant de la 
gauche radicale, qui souhaitaient réaliser une communauté conforme aux principes du socialisme et susceptible, en quelque sorte, de dénoncer le régime politique en place. En application du principe égalitaire, chaque nouvel arrivant s'est vu attribuer une surface identique pour s'installer (Erdi-Lelandais, 2014). L'un des premiers habitants du 1er-Mai, figure emblématique du quartier, nous précise le contexte et les modalités de cette opération : «Nous avons appris par la famille, les connaissances et les amis que les socialistes construisaient des gecekondus près des carrières à Ümraniye. J'ai décidé d'y aller avec mon frère. Au départ, c'était une zone près des déchetteries. Il y avait partout des potagers, pas une seule route, ni électricité, ni eau. Nous avons décidé de construire une cabane. Les révolutionnaires nous ont aidés. Ils participaient à la construction » (Entretien avec S.K., 5 avril 2011).

Quelque temps après, les gecekondus s'étant multipliés, les groupes contrôlant les installations commencèrent à réaliser quelques équipements, tels une maison du peuple ou un espace santé. Toutefois, en raison de l'illégalité des constructions et de celle du quartier tout entier, les habitants étaient régulièrement confrontés à des tentatives de démolition de la part des autorités municipales. La défense du quartier par ces habitants face à ces tentatives a atteint son paroxysme le 2 septembre 1977, date à laquelle cinq habitants ont perdu leur vie lors d'affrontements. À partir de cette date, la municipalité a abandonné sa volonté de démolition.

À Ankara, le quartier de la Vallée de Dikmen partage avec celui du 1er-Mai à Istanbul une histoire assez proche, mais il s'en distingue par le fait que les groupements politiques qui furent à l'origine de sa création se sont, par la suite, progressivement affaiblis. Sans surprise cependant, ce sont les classes défavorisées et à faibles revenus, occupant des emplois précaires dans le secteur informel, qui y dominent toujours largement.

Les premières constructions de la Vallée de Dikmen ont été réalisées à peu près au même moment qu'au 1er-Mai et dans un contexte assez semblable, ce qui ressort des propos de Tarik :

"Je suis arrivé d'Erzincan à Ankara en 1973. Mon cousin m'a trouvé du travail comme gardien dans le campus de l'Université technique du Moyen-Orient. Il y avait une grande lutte politique à l'époque dans cette Université. J'y ai participé et commencé à apprendre des choses, notamment ma conscience de classe. Après avoir participé à la lutte, on s'est demandé : "Que peut-on faire pour les nouveaux arrivants d'Anatolie sans emploi, sans logis ?" On a cherché où il y avait des terrains publics à Ankara. On s'est mis ensemble à cinq amis et nous avons trouvé la vallée de Dikmen [...]» (Entretien du 29 octobre 2013).

En raison de l'augmentation rapide de la population et du nombre de gecekondus dans la Vallée de Dikmen, les municipalités et les gouvernements successifs ont finalement été contraints d'y assurer certains services publics : ouverture d'une ligne d'autobus, goudronnage des rues, branchement au réseau d'électricité, etc. Cette reconnaissance de fait procure aux habitants un sentiment de plus grande sécurité, favorise leur stabilité et leur permet de pouvoir progressivement s'insérer dans la ville. Une partie des habitants est même parvenue, dans le courant des années 1980, à obtenir de la municipalité la délivrance de certificats de droit d'occupation (tapu tahsis senedi); cela ne concernait toutefois qu'environ un tiers d'entre eux.

Les programmes extrêmement ambitieux de rénovation urbaine, lancés par le gouvernement de l'AKP dès 2005, ont des objectifs multiples : éradiquer les quartiers de gecekondus, assainir le marché du logement, faire en sorte que les nouvelles constructions 
puissent résister aux tremblements de terre, etc ${ }^{7}$. Mais l'éradication des constructions insalubres et, plus largement, des quartiers de gecekondus, dont la plupart sont passés progressivement d'une localisation périphérique à une situation péricentrale, fournit aussi des opportunités foncières et des possibilités de plus-values inestimables aux promoteurs immobiliers.

Concernant ces deux quartiers, de nombreux projets de démolition et d'expropriation ont donc été lancés, sans que les habitants en aient été officiellement informés au préalable et qu'une concertation ait été engagée avec eux. Ces derniers les perçoivent comme une menace d'autant plus grande qu'ils étaient parvenus, peu à peu, à s'ancrer dans leur quartier et à s'insérer dans la ville au point qu'ils y pensaient leur place acquise. De nouveaux projets de transformation urbaine visant la démolition totale de nombreux quartiers provoquent donc des réactions de la part des habitants qui se sont accrochés à la vie urbaine tant bien que mal malgré l'absence des politiques de logement social et de service public au sein de leur quartier. Cela a été le cas dans les deux quartiers que nous venons de présenter. L'étude de leurs mobilisations mériterait sans nul doute de faire l'objet d'un autre article, mais, même sans les traiter de façon développée, elles en disent long sur la trajectoire des migrants ruraux dans les grandes métropoles turques, ainsi que sur les politiques d'urbanisation en Turquie qui s'inscrivent dans une tendance globale des politiques en Turquie qui est l'autoritarisme de plus en plus accrue de leur conception et mise en œuvre.

\section{En guise de conclusion}

Les habitants des gecekondus sont vulnérables, mais ils n'en disposent pas moins toujours de ressources mobilisables. L'expansion et les caractéristiques des gecekondus peuvent donc être analysées sous l'angle de la vulnérabilité résidentielle. Les exemples ici étudiés illustrent comment les habitants, citadins de fraîche date au début du processus, en situation de vulnérabilité, inventent des moyens et mobilisent leurs compétences, malgré leurs moyens limités, pour pérenniser leur place dans la ville et préserver cette place une fois qu'ils l'ont gagnée (Erdi 2019). Pour parvenir à cet objectif, des résistances se forgent parfois et s'unissent dans un objectif d'émancipation partagé, ce qui leur permet de générer une identité commune. Leurs adversaires, qui sont aussi potentiellement leurs interlocuteurs, s'organisent également et affinent leurs stratégies. Au cours du temps, les agents de l'État tentent par exemple de diviser les habitants en leur offrant soit des avantages individuels (un poste à la mairie, une place dans la représentation politique, etc.), soit des compensations financières. Au bout du compte, dans le cas de Dikmen, la durée très longue de la résistance des habitants - plus de huit années - a contraint la mairie à négocier des conditions plus favorables aux résidents pour éviter le blocage du projet et éviter une perte d'argent. Dans le cas du 1er-Mai, les autorités municipales hésitent pour l'instant à lancer un projet de grande ampleur, car ils se méfient des réactions des habitants du quartier, dont le passé militant leur laisse craindre un conflit de longue durée (Erdi 2019).

Afin de faciliter les démolitions et les déplacements, la transformation urbaine vise les populations pauvres et l'économie informelle tout en procédant à un maintien renforcé de l'ordre, avec la privatisation de la sécurité (à titre d'exemple, l'accès à toutes les Universités est réglementé par des équipes privées de sécurité) et la (ré)émergence d'un discours souvent racialisé et criminalisant des pauvres étiquetés comme dangereux et criminels. La municipalité

\footnotetext{
${ }^{7}$ De ce fait, des démolitions de gecekondus, auparavant rares à Istanbul, se sont multipliées d'une manière spectaculaire. Au cours de la période allant de 2004 à 2008, 11543 unités ont été démolies à Istanbul, un record depuis l'apparition des gecekondus dans les années 1950 (Kuyucu, Ünsal, 2010: 1484).
} 
de Küçükçekmece, sur le territoire de laquelle se trouve le projet de transformation du quartier Ayazma dont la majorité des habitants sont des Kurdes, justifie ainsi son action : « Il est essentiel de réaménager certains endroits dans la ville - qui sont problématiques, insalubres, socialement corrompus, des lieux de crime et manquant de qualité urbaine et de sécurité humaine - comme des lieux sains et vivables » (K. Municipality 2007: 6).

Au bout du compte, depuis maintenant plus de 10 ans, l'urbanisation en Turquie suit une tendance de plus en plus autoritaire et elle est symptomatique d'un système politique global engagé depuis quelques années dans une direction autoritaire. Ces politiques impliquent des stratégies nouvelles et agressives de maintien de l'ordre et de surveillance des groupes et espaces particuliers, la criminalisation de la pauvreté et l'utilisation accrue du système pénal (Dikeç, 2009). Pour la première fois en 2004, l'adoption d'un nouveau Code pénal (loi ${ }^{\circ}{ }^{\circ}$ 5237) fait de la construction d'un gecekondu une infraction pénale, passible de cinq ans de prison. Cela révèle clairement l'approche «tolérance zéro » du gouvernement sur cette question, mais démontre également une absence de volonté de sa part d'apporter des solutions pérennes et démocratiques au problème de logement de ses populations.

\section{Références}

Adanalı Y., Korkmaz T., Yücesoy E.Ü. 2009. Living in Voluntary and Involuntary Exclusion, Istanbul: Refuge-Diwan, available on http://reclaimistanbul.com/2011/04/04/istanbul-livingin-exclusion/

Bekmen A. (2014) 'State and Capital in Turkey During the Neoliberal Era', in Akça I., Bekmen A., Özden B.A. (ed.), Turkey Reframed. Constituting Neoliberal Hegemony, London: Pluto Press. Bekmen A. 2014. "State and Capital in Turkey During the Neoliberal Era", in Akça I., Bekmen A., Özden B.A. (ed.), Turkey Reframed. Constituting Neoliberal Hegemony, London: Pluto Press. Brodiez-Dolino A. 2016. «Le concept de vulnérabilité », La Vie des idées, 11 février 2016, URL: http://www.laviedesidees.fr/Le-concept-de-vulnerabilite.html, consulté le 2 juin 2018.

Çavuşoglu E. et Strutz J. 2014. "'We'll Come and Demolish Your House !' : The Role of Spatial (Re)Production in the Neoliberal Hegemonic Politics of Turkey » in Akça I., Bekmen A., Özden B.A. (ed.), Turkey Reframed. Constituting Neoliberal Hegemony, London: Pluto Press.

Dikeç, M. 2009. Space, politics and (in)justice / L'espace, le politique et l'injustice. Justice spatiale | Spatial justice, $\mathrm{n}^{\circ}$. 1, September; available at http://www.jssj.org.

Erdi G. 2016. «Les politiques urbaines en Turquie : entre conservatisme néolibéral et autoritarisme », Métropolitiques, 20 juin 2016. URL : https://metropolitiques.eu/Les-politiques-urbaines-enTurquie-entre-conservatisme-neoliberal-et.html

Erdi G. 2019. «Quartiers informels au prisme de la vulnérabilité résidentielle en Turquie », in Deboulet A., Fijalkow Y., Bouillon F. (dir.), Vulnérabilités résidentielles, Paris, L’Aube.

Florida R. 2002. The Rise of the Creative Class--Revisited: Revised and Expanded [2014], NY : Basic Book.

Florida R. 2017. The new urban crisis: how our cities are increasing inequality, deepening segregation, and failing the middle class - and what we can do about it, NY : Basic Books.

Genard, J-L. 2014. « La question de la responsabilité sous 1'horizon du référentiel humanitaire », in Axelle Brodiez-Dolino, Isabelle von Bueltzingsloewen, Benoît Eyraud, Christian Laval et 
Bertrand Ravon (dir.), Vulnérabilités sanitaires et sociales. De l'histoire à la sociologie, Rennes, PUR.

Gürek, H. 2008. AKP'nin Müteahhitleri (Constructors of the AKP), Istanbul: Güncel.

Karpat K. 1976. The Gecekondu. Rural Migration and Urbanization. Cambridge, Cambridge University Press.

Keleş R. 1992. Yerinden Yönetim ve Siyaset (Decentralization and Politics). Istanbul: Cem.

Küçükçekmece Belediyesi. 2007. Ayazma-Tepeüstü Kentsel Dönüşüm Projesi; Yaşama Yeni Bir Pencereden Bakın (Ayazma-Tepeüstü Urban Transformation Project: Watch the life from a new window), Istanbul: Küçükçekmece Belediyesi.

Lees L., Slater T., Wyly E. 2010. The Gentrification Reader, London : Routledge.

Öncü, A. (1988). «The politics of urban land market in Turkey: 1950-1980», International Journal of Urban and Regional Research 12.1, 38-64.

Smith N. 1979. «Toward a Theory of Gentrification A Back to the City Movement by Capital, not People », Journal of the American Planning Association, 45:4, p.538-548.

Smith N. 1996. The New Urban Frontier: Gentrification and the Revanchist City, London : Routledge. TUIK-Institut national des statistiques de Turquie.

Zukin S. 1978. «Gentrification: Culture and Capital in the Urban Core », Annual Review of Sociology, 13:1, p.129-147. 